\title{
THE EFFECT OF INITIAL MOISTURE CONTENT, PACKAGING AND STORAGE PERIOD ON SUCCINATE DEHYDROGENASE AND CYTOCHROME OXIDASE ACTIVITY OF SOYBEAN SEED
}

\author{
AURELLIA TATIPATA \\ Department of Agronomy, Faculty of Agriculture, Pattimura University, \\ Ambon 97000, Indonesia
}

\begin{abstract}
The quality of soybean seeds decreased rapidly, so it would be difficult to keep them for a long time. This research was aimed to study the effect of initial moisture content, packaging material and storage period on succinate dehydrogenase and cytochrome oxidase activities. The experimental design used was Factorial Randomized Complete Block Design (RCBD), consisting of three factors, i.e. moisture content (8.10 and 12 percent), packaging materials (polyethylene, wheat and aluminium foil), storage period ( $0,1,2,3,4,5$, and 6 months). Changes in seed water contents, specific activities of succinate dehydrogenase and cytochrome oxidase, germination and coefficients velocity of germination, were monthly determined. It was found that decrease of succinate dehydrogenase specific activity can be induced if initial seed water content is raised above $8 \%$. Soybean seeds stored in aluminium foil bags showed high succinate dehydrogenase and cytochrome oxidase specific activities, viability, coefficient of velocity of germination and could maintain the seeds at a low moisture content to delay seed deterioration compared to seeds stored in polyethylene and wheat bags.
\end{abstract}

Keywords: moisture content, succinate dehydrogenase, cytochrome oxidase, soybean

\section{INTRODUCTION}

Soybean seeds are usually stored for about three months before planting. The viability of soybean seeds in the sub-humid tropics such as Indonesia will decrease during storage. This is one of the factors which inhibit soybean seed production.

Soybean seeds may loose its viability or deteriorate even in three months if kept at $14 \%$ moisture content and $30^{\circ} \mathrm{C}$ (Sadjad 1980). According to Sumarno and Harnoto (1983), viability of soybean seeds decreased to $57 \%$ after 6 months of storage if kept at above $13 \%$ moisture content, $20^{\circ} \mathrm{C}$, and relative humidity of $50 \%$. Viability of

Coresponding author: lethatatipata@yahoo.com 
soybean seeds may decrease to as much as $50 \%$ if kept at 27 to $32^{\circ} \mathrm{C}$ and relative humidity of $80 \%$. When longer storage is needed, seed moisture content should be less than $11 \%$, temperature of $20^{\circ} \mathrm{C}$ and relative humidity of $50 \%$, and the seeds should be packed in moisture resistant containers (Wilson \& McDonald 1992). Soybean seeds could be safely stored for 10 months using vacuum plastic bags with initial moisture content of around 8\% (Harnowo \& Adie 1998). Viability of soybean seeds that were stored at $8 \%$ initial moisture content at room temperature for 3 years did not decrease, but it was $60 \%$ after 1 month if stored at $12 \%$ and $0 \%$ after three months of storage (Kartono 2004). Soybean seeds are hygroscopic, so $\mathrm{H}_{2} \mathrm{O}$ is absorbed from the environment.

Polyethylene and aluminium foil materials were moderately effective in preventing moisture uptake and maintaining seed viability, while paper and cloth containers were less effective (Wilson \& McDonald 1992).

Seed deterioration is an inexorable and irreversible process. One of the symptoms of seed deterioration is the decrease of the enzyme activities such as succinate dehydrogenase and cytochrome oxidase (Copeland \& McDonald 1985). However no detailed studies are available concerning the possible relationship between the activities of succinate dehydrogenase and cytochrome oxidase and the viability of soybean seeds.

The aim of this research was to study the effects of initial water content of seeds, packaging materials and storage periods on succinate dehydrogenase and cytochrome oxidase activities of soybean seeds.

The hypotheses of this study were: (1) succinate dehydrogenase and cytochrome oxidase specific activities of stored soybean seeds did not decrease at $8 \%$ moisture content; (2) succinate dehydrogenase and cytochrome oxidase specific activities of soybean seeds that were packed in aluminium foil bags did not decrease during 6 months of storage; (3) succinate dehydrogenase and cytochrome oxidase activities of soybean seeds that were stored at $8 \%$ initial moisture content in aluminium foil bags did not decrease during 6 months of storage.

\section{MATERIALS AND METHODS}

\section{Sample collection}

Soybean seeds var. Willis were obtained in March 2007 from Balai Benih Induk Wonosari Yogyakarta. After seed processing, water contents of seeds were adjusted to $8 \%, 10 \%$, and $12 \%$. Samples of $500 \mathrm{~g}$ were placed in polyethylene plastic bags, wheat bags and aluminium foil bags for 6 months at room temperature. Storage temperature and relative humidity were monitored throughout the experiment but were not controlled. One sample from each treatment was taken monthly. All samples were tested after storage for seed moisture content, specific activities of succinate dehydrogenase and cytochrome oxidase, viability, and coefficient velocity of germination. Viability and coefficient velocity of germination of seed before storage were $100 \%$. 


\section{Isolation and purification of mitochondria}

Mitochondria were isolated from $3 \mathrm{~g}$ of 3 -d-old soybean hypocotyls by the procedure of Day and Hanson (1977), modified by using the centrifugation speeds of Beckman-J6B centrifuge. Samples were kept in a refrigerator for 10 minutes at $10^{\circ} \mathrm{C}$ before being grounded by hand with a chilled mortar and pestle. The filtered homogenates were centrifuged at $400 \times g, 500 \times g$ and $600 \times g$ for 10 minutes, respectively. The pellets (containing cell walls, fragments, starch grains, nuclei, and intact plastids) were discarded. The supernatants were then centrifuged at $10000 \times \mathrm{g}$ for 20 minutes and mitochondria pellets were resuspended in $150 \mu$ of a reaction mixture consisting of $0.4 \mathrm{M}$ sucrose, $0.5 \%$ (w/v) bovine serum albumin (BSA), $50 \mathrm{mM}$ Tris $\mathrm{pH} 7.6$ and $10 \mathrm{mM} \mathrm{KH}_{2} \mathrm{PO}_{4}$. Triplicate aliquots $(10 \mu \mathrm{l})$ each of the resuspension were saved for protein determination (corrected for added BSA). Protein contents in the mitochondria suspension dissolved in $1 \mathrm{M} \mathrm{NaOH}$ were measured according to Lowry et al. (1951). The mitochondria was sonicated until the suspension cleared (about 20 seconds) at 20 kilocycle using sonicator Labsonic U. Mitochondria inner membranes were pelleted by centrifugation for 20 minutes at $100000 \times g$ (Hayes et al. 1991) using Hitachi SCP 85H ultracentrifuge. The pellets were resuspended in $5 \mathrm{ml}$ of buffer Tris $\mathrm{pH} 7.6$ and stored at $0^{\circ} \mathrm{C}$ until further processing.

\section{Enzyme assays}

All enzyme activities were measured at $25^{\circ} \mathrm{C}$ and the neutral linear rates were measured. Cytochrome oxidase activity was measured according to Nawa and Asahi (1971).

The activity of cytochrome oxidase was assayed with the medium $(2.5 \mathrm{ml})$ containing $1 \mu \mathrm{M}$ horse heart cytochrome c, $50 \mu \mathrm{M}$ phosphate buffer ( $\mathrm{pH} 7.2), 250 \mu \mathrm{M}$ N,N,N,N-tetramethyl-p-phenylenediamin, $1 \mathrm{mM}$ ascorbic acid and $35 \mu \mathrm{l}$ inner membrane mitochondria suspension. This activity was measured as the change in absorbance at $550 \mathrm{~nm}$ using a Beckman 1601 spectrophotometer with the dual wavelength mode and an extinction coefficient of $17.6 \mathrm{mM}^{-1} \mathrm{~cm}^{-1}$ for 1.5 minutes with interval of 15 seconds at room temperature $\left(21^{\circ} \mathrm{C}\right)$. Specific activity of cytochrome oxidase was $\mu \mathrm{mol}$ menit ${ }^{-1} \mathrm{mg}$ protein ${ }^{-1}$. Protein content was determined by the Lowry method (1951). BSA concentrations as standard solution were 0, 0.1, 0.2, 0.4, 0.6, 0.8 and $1.0 \mathrm{mgml}^{-1}$.

Succinate dehydrogenase activity was studied using a solution $(2.5 \mathrm{ml})$ containing $20 \mathrm{mM}$ phosphate buffer (pH 7.6), $1 \mathrm{mM} \mathrm{KCN,} \mathrm{10 \%} \mathrm{Triton} \mathrm{X-100,} 0.1$ mM EDTA, 50 $\mu \mathrm{l}$ of inner membrane mitochondria suspension (Singer et al. 1972). This activity was measured as the change in absorbance at $600 \mathrm{~nm}$ using a Beckman UV-Vis Shimadzu 1601 spectrophotometer with the dual wavelength mode and an extinction coefficient of $19.6 \mathrm{mM}^{-1} \mathrm{~cm}^{-1}$ for 1 minute with interval of 10 seconds at room temperature

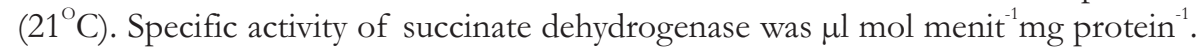
Protein content was determined by Lowry et al. method (1951) with BSA concentrations as standard of $0,0.1,0.2,0.4,0.6,0.8$ and $1.0 \mathrm{mg} \mathrm{ml}^{-1}$.

Protein content was assayed with a medium $(2 \mathrm{ml})$ containing $1 \mathrm{ml}$ of $2 \%(\mathrm{w} / \mathrm{v})$ $\mathrm{Na}_{2} \mathrm{CO}_{3}, 0.1 \mathrm{~N} \mathrm{NaOH}, 2 \%(\mathrm{w} / \mathrm{v}) \mathrm{Na}^{+} \mathrm{K}^{+}$tartrate and $1 \%$ (w/v) $\mathrm{CuSO}_{4} .5 \mathrm{H}_{2} \mathrm{O}$ with 
comparison of 10:0.5:0.5. After $3 \mathrm{ml}$ of folin-ciocalteau was added, it was incubated for 10 minutes. Absorbance was measured at $600 \mathrm{~nm}$. BSA solutions as standard were $0,0.1,0.2,0.4,0.6,0.8$ and $1.0 \mathrm{mgml}^{-1}$.

\section{Germination and vigor test}

All samples were tested for standard germination and vigor (coefficient velocity of germination). A number of 100 seeds with 4 replications were germinated in a petri dish where a paper wetted with distilled $\mathrm{H}_{2} \mathrm{O}$ was used as underlayer. The number of seeds germinated (embryonic axis were longer than $1 \mathrm{~cm}$ ) was recorded daily for 7 days

Seed germination quantities were determined according to International Seed Testing Association (1996) using the following formula :

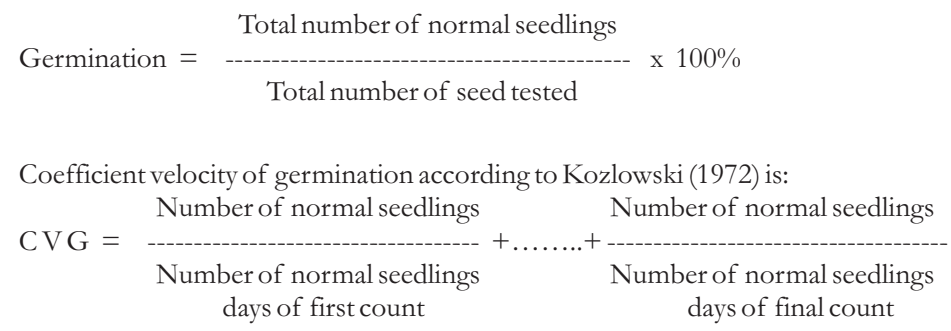

\section{Statistical analysis}

The experimental design used was Factorial Experiment in Randomized Complete Block Design (RCBD) with three factors and three replications. Those factors are initial moisture content (A) which consisted of 3 levels $8 \%, 10 \%, 12 \%$; packaging materials which consisted of 3 kinds (polyethylene plastic bag, wheat bag, aluminium foil bag); storage period which consisted of 7 levels 0 (control), 1 month, 2 months, 3 months, 4 months, 5 months, and 6 months. Data were analyzed using analysis of variance of Statistical Analyses System (SAS 1986). Regression analysis and Pearson correlation coefficients were done to determine relationships between initial seed moisture contents and seed moisture contents after storage, germinations, coefficient velocity of germination (CVG), specific activity of succinate dehydrogenase and specific activity of cytochrome oxidase.

\section{RESULTS AND DISCUSSIONS}

Interactions among initial seed water contents, packaging materials and storage periods on seed water content, specific activity of succinate dehydrogenase, germination and coefficient velocity of germination after storage are shown in Tables 1, 2, 4 and 5. The water contents of seeds after storage at all levels of initial moisture contents of seeds stored in wheat bag increased faster than other packaging materials, because wheat bags were not moisture proof compared to polyethylene and aluminium foil bags. Correlation between initial moisture content of seeds and seed 
moisture contents after storage was positive (Fig. 1). The seed lots stored in wheat bags after 2 months showed an increase in moisture content of $13.03 \%$ causing a decrease in specific activity of succinate dehydrogenase (1,34 Umgprotein ${ }^{-1}$ ) (Table 2 and Fig. 2). This indicates that initial moisture content of seeds, packaging materials

Table 1. The effect of initial moisture content, packaging materials and storage period on seed moisture conten $(\%)$

\begin{tabular}{|c|c|c|c|c|}
\hline \multirow{2}{*}{$\begin{array}{c}\text { Initial } \\
\text { moisture } \\
\text { content } \\
(\%) \\
\end{array}$} & \multirow{2}{*}{$\begin{array}{c}\text { Storage } \\
\text { period } \\
\text { (month) }\end{array}$} & \multicolumn{3}{|c|}{ Packaging materials } \\
\hline & & $\begin{array}{l}\text { Polyethylene } \\
\text { (B1) }\end{array}$ & $\begin{array}{l}\text { Wheat } \\
\text { (B2) }\end{array}$ & $\begin{array}{c}\text { Alfoil } \\
\text { (B3) }\end{array}$ \\
\hline \multirow[t]{7}{*}{$8\left(A_{1}\right)$} & $\mathrm{C}_{0}(0)$ & $8.00 \mathrm{q}-\mathrm{r}$ & $8.00 \mathrm{q}-\mathrm{r}$ & $8.00 \mathrm{q}-\mathrm{r}$ \\
\hline & $\mathrm{C}_{1}(1)$ & $8.03 \mathrm{q}-\mathrm{r}$ & $8.67 \mathrm{p}-\mathrm{r}$ & $8.00 \mathrm{q}-\mathrm{r}$ \\
\hline & $\mathrm{C}_{2}(2)$ & 8.63 p-r & $9.24 \mathrm{~m}-\mathrm{q}$ & $8.63 \mathrm{p}-\mathrm{r}$ \\
\hline & $C_{3}(3)$ & $8.70 \mathrm{p}-\mathrm{r}$ & $9.20 \mathrm{~m}-\mathrm{q}$ & $8.70 \mathrm{o}-\mathrm{r}$ \\
\hline & $\mathrm{C}_{4}(4)$ & $8.87 \mathrm{o}-\mathrm{r}$ & $11.23 \mathrm{~b}-\mathrm{i}$ & 8.84 o-r \\
\hline & $\mathrm{C}_{5}(5)$ & 8.98 o-r & $11.40 \mathrm{~b}-\mathrm{h}$ & $8.92 \mathrm{o}-\mathrm{r}$ \\
\hline & $\mathrm{C}_{6}(6)$ & $11.24 \mathrm{~b}-\mathrm{i}$ & $11.96 \mathrm{~b}-\mathrm{e}$ & $10.20 \mathrm{f}-\mathrm{n}$ \\
\hline \multirow[t]{7}{*}{$10\left(\mathrm{~A}_{2}\right)$} & $\mathrm{C}_{0}(0)$ & $10.00 \mathrm{~h}-\mathrm{p}$ & 10.00 h-p & $10.00 \mathrm{~h}-\mathrm{p}$ \\
\hline & $C_{1}(1)$ & $10.15 \mathrm{f}-\mathrm{n}$ & $10.35 \mathrm{f}-\mathrm{n}$ & $10.00 \mathrm{~h}-\mathrm{p}$ \\
\hline & $\mathrm{C}_{2}(2)$ & $10.23 \mathrm{f}-\mathrm{n}$ & $10.63 \mathrm{e}-\mathrm{m}$ & $10.181-\mathrm{p}$ \\
\hline & $\mathrm{C}_{3}(3)$ & $10.66 \mathrm{e}-\mathrm{m}$ & $11.00 \mathrm{~b}-\mathrm{k}$ & $10.22 \mathrm{f}-\mathrm{n}$ \\
\hline & $\mathrm{C}_{4}(4)$ & $10.72 \mathrm{c}-1$ & $11.48 \mathrm{~b}-\mathrm{f}$ & $10.33 \mathrm{f}-\mathrm{n}$ \\
\hline & $\mathrm{C}_{5}(5)$ & $10.75 \mathrm{~d}-1$ & $11.60 \mathrm{~b}-\mathrm{j}$ & $10.60 \mathrm{e}-\mathrm{m}$ \\
\hline & $\mathrm{C}_{6}(6)$ & $11.81 \mathrm{~b}-\mathrm{e}$ & $12.40 \mathrm{~b}$ & $11.25 \mathrm{~b}-\mathrm{i}$ \\
\hline \multirow[t]{7}{*}{$12\left(\mathrm{~A}_{3}\right)$} & $\mathrm{C}_{0}(0)$ & $12.00 \mathrm{~b}-\mathrm{e}$ & $12.00 \mathrm{~b}-\mathrm{e}$ & $12.00 \mathrm{~b}-\mathrm{e}$ \\
\hline & $\mathrm{C}_{1}(1)$ & $12.12 \mathrm{~b}-\mathrm{d}$ & $12.42 \mathrm{~b}$ & $12.00 \mathrm{~b}-\mathrm{e}$ \\
\hline & $\mathrm{C}_{2}(2)$ & $12.22 \mathrm{~b}-\mathrm{c}$ & $12.64 \mathrm{a}-\mathrm{b}$ & $12.14 \mathrm{~b}-\mathrm{d}$ \\
\hline & $\mathrm{C}_{3}(3)$ & $12.25 \mathrm{~b}-\mathrm{c}$ & $13.03 \mathrm{c}$ & $12.18 \mathrm{~b}-\mathrm{c}$ \\
\hline & $\mathrm{C}_{4}(4)$ & $12.36 \mathrm{~b}$ & $13.26 \mathrm{a}$ & $12.24 \mathrm{~b}-\mathrm{c}$ \\
\hline & $\mathrm{C}_{5}(5)$ & $12.42 \mathrm{~b}$ & $13.42 \mathrm{a}$ & $12.26 \mathrm{~b}-\mathrm{c}$ \\
\hline & $\mathrm{C}_{6}(6)$ & $12.50 \mathrm{a}-\mathrm{c}$ & $13.58 \mathrm{a}$ & $12.28 \mathrm{~b}$ \\
\hline
\end{tabular}

Note: Numbers followed by the same alphabet are not significantly different at $5 \%$ of IMRI

$\mathrm{h}-\mathrm{p}=$ hijklmnop
Table 2. The effect of initial moisture content, packaging materials and storage period on specific activity of succinate dehydrogenase ((Umg protein $\left.{ }^{-1}\right)$ (transformation of $\mathrm{X}+0.5)^{0.5}$

\begin{tabular}{|c|c|c|c|c|}
\hline \multirow{2}{*}{$\begin{array}{c}\text { Initial } \\
\text { moisture } \\
\text { content } \\
(\%) \\
\end{array}$} & \multirow{2}{*}{$\begin{array}{l}\text { Storage } \\
\text { period } \\
\text { (month) }\end{array}$} & \multicolumn{3}{|c|}{ Packaging materials } \\
\hline & & $\begin{array}{l}\text { Polyethylene } \\
\text { (B1) }\end{array}$ & $\begin{array}{l}\text { Wheat } \\
\text { (B2) }\end{array}$ & $\begin{array}{l}\text { Alfoil } \\
\text { (B3) }\end{array}$ \\
\hline \multirow[t]{7}{*}{$8\left(A_{1}\right)$} & $\mathrm{C}_{0}(0)$ & $3.39 \mathrm{a}-\mathrm{b}$ & $3.00 \mathrm{a}-\mathrm{c}$ & $3.94 \mathrm{a}$ \\
\hline & $\mathrm{C}_{1}(1)$ & $3.09 \mathrm{a}-\mathrm{c}$ & $1.73 \mathrm{c}-\mathrm{g}$ & $3.60 \mathrm{a}$ \\
\hline & $\mathrm{C}_{2}(2)$ & $2.84 \mathrm{a}-\mathrm{c}$ & $1.63 \mathrm{c}-\mathrm{h}$ & $2.79 \mathrm{a}-\mathrm{d}$ \\
\hline & $C_{3}(3)$ & $2.79 \mathrm{a}-\mathrm{d}$ & $1.60 \mathrm{c}-\mathrm{h}$ & $2.78 \mathrm{a}-\mathrm{d}$ \\
\hline & $\mathrm{C}_{4}(4)$ & $2.52 \mathrm{a}-\mathrm{e}$ & $1.37 \mathrm{~d}-\mathrm{h}$ & $2.59 \mathrm{a}-\mathrm{e}$ \\
\hline & $\mathrm{C}_{5}(5)$ & $2.07 \mathrm{~b}-\mathrm{g}$ & $1.13 \mathrm{e}-\mathrm{h}$ & $2.39 \mathrm{~b}-\mathrm{f}$ \\
\hline & $\mathrm{C}_{6}(6)$ & $0.97 \mathrm{f}-\mathrm{h}$ & $0.89 \mathrm{f}-\mathrm{h}$ & $1.60 \mathrm{c}-\mathrm{h}$ \\
\hline \multirow[t]{7}{*}{$10\left(\mathrm{~A}_{2}\right)$} & $\mathrm{C}_{0}(0)$ & $2.52 \mathrm{a}-\mathrm{e}$ & $2.84 \mathrm{a}-\mathrm{c}$ & $2.79 \mathrm{a}-\mathrm{d}$ \\
\hline & $C_{1}(1)$ & $2.07 \mathrm{~b}-\mathrm{g}$ & $2.00 \mathrm{~b}-\mathrm{g}$ & $2.14 \mathrm{~b}-\mathrm{g}$ \\
\hline & $\mathrm{C}_{2}(2)$ & $1.98 \mathrm{~b}-\mathrm{g}$ & $1.88 \mathrm{~b}-\mathrm{g}$ & $1.97 \mathrm{~b}-\mathrm{g}$ \\
\hline & $C_{3}(3)$ & $1.54 \mathrm{c}-\mathrm{h}$ & $1.34 \mathrm{~d}-\mathrm{h}$ & $1.64 \mathrm{c}-\mathrm{h}$ \\
\hline & $\mathrm{C}_{4}(4)$ & $1.37 \mathrm{~d}-\mathrm{h}$ & $1.17 \mathrm{e}-\mathrm{h}$ & $1.38 \mathrm{~d}-\mathrm{h}$ \\
\hline & $\mathrm{C}_{5}(5)$ & $1.04 \mathrm{e}-\mathrm{h}$ & $1.01 \mathrm{e}-\mathrm{h}$ & $1.18 \mathrm{e}-\mathrm{h}$ \\
\hline & $\mathrm{C}_{6}(6)$ & $0.95 \mathrm{f}-\mathrm{h}$ & $0.09 \mathrm{~h}$ & $0.98 \mathrm{e}-\mathrm{h}$ \\
\hline \multirow[t]{7}{*}{$12\left(\mathrm{~A}_{3}\right)$} & $\mathrm{Co}_{0}(0)$ & $2.81 \mathrm{a}-\mathrm{c}$ & $2.80 \mathrm{a}-\mathrm{c}$ & $2.83 \mathrm{a}-\mathrm{c}$ \\
\hline & $\mathrm{C}_{1}(1)$ & $1.99 \mathrm{~b}-\mathrm{g}$ & $1.83 \mathrm{~b}-\mathrm{g}$ & $2.03 \mathrm{~b}-\mathrm{g}$ \\
\hline & $\mathrm{C}_{2}(2)$ & $1.59 \mathrm{c}-\mathrm{h}$ & $1.49 \mathrm{c}-\mathrm{h}$ & $1.62 \mathrm{c}-\mathrm{h}$ \\
\hline & $\mathrm{C}_{3}(3)$ & $1.50 \mathrm{c}-\mathrm{h}$ & $1.34 \mathrm{~d}-\mathrm{h}$ & $1.55 \mathrm{c}-\mathrm{h}$ \\
\hline & $\mathrm{C}_{4}(4)$ & $1.12 \mathrm{e}-\mathrm{h}$ & $1.05 \mathrm{e}-\mathrm{h}$ & $1.34 \mathrm{~d}-\mathrm{h}$ \\
\hline & $\mathrm{C}_{5}(5)$ & $1.05 \mathrm{e}-\mathrm{h}$ & $0.90 \mathrm{f}-\mathrm{h}$ & $1.29 \mathrm{~d}-\mathrm{h}$ \\
\hline & $\mathrm{C}_{6}(6)$ & $0.97 \mathrm{f}-\mathrm{h}$ & $0.75 \mathrm{~g}-\mathrm{h}$ & $0.98 \mathrm{e}-\mathrm{h}$ \\
\hline
\end{tabular}

Note: Numbers followed by the same alphabet are not significantly different at $5 \%$ of DMRI $\mathrm{e}-\mathrm{h}=\mathrm{efgh}$

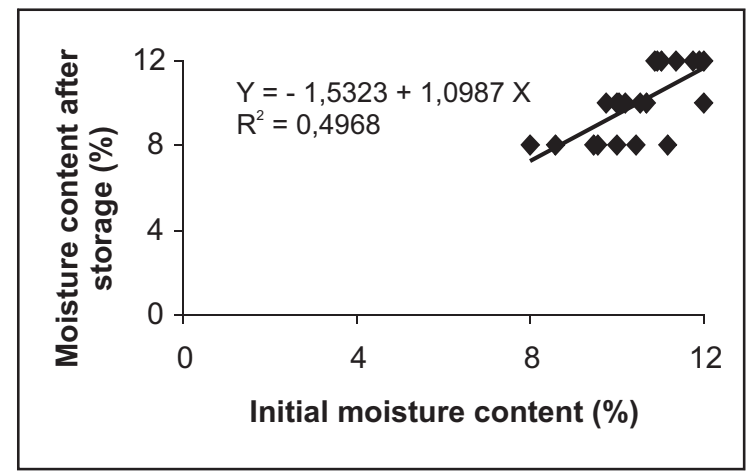

Figure 1. Relationship between initial moisture content and seed moisture content after storage 


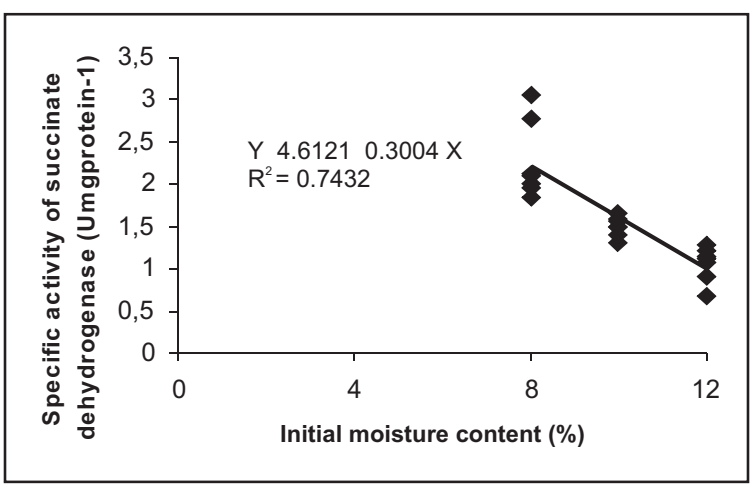

Figure 2. Relationship between initial moisture and specific activity of succinate dehydrogenase

and storage periods are the three principal factors which affect the activity of dehydrogenase enzyme and viability of seeds during storage. According to Harrington (1959), the two most important environmental factors which influence the speed of seed aging are the relative humidity of the air and temperature. Relative humidity of the air controls seed moisture content, while temperature affects the rates of biochemical processes in seeds. Harrington concluded that for every increase in seed moisture content of $1 \%$ and $5{ }^{\circ} \mathrm{C}$ in temperature, the storage life of seed becomes halved.

Cytochrome oxidase specific activity of stored soybean seeds at all levels of initial moisture did not significantly decrease (Table 3 ). This activity decreased because moisture content of soybean seeds increased (Fig. 3 and Fig. 4). Higher moisture content of seeds cause a decrease of inner membrane integrity of mitochondria. It has been clearly demonstrated that mitochondria activity was the primary source of energy during seed germination (Attuci et al. 1991). Succinate dehydrogenase is located on the inner surface of mitochondria inner membrane (Moller \& Rein 1986) which has a lower affinity for NADH.

Table 3. The effect of initial moisture contents and storage period on specific activity of cytochrome oxidase

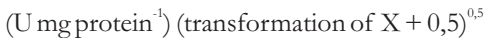

\begin{tabular}{|c|c|c|c|}
\hline \multirow{2}{*}{ Storage period(month) } & \multicolumn{3}{|c|}{ Initial Moisture content $(\%)$} \\
\hline & 8 & 10 & 12 \\
\hline 0 & $0.7077 \mathrm{~b}$ & $0.7082 \mathrm{~b}$ & $0.8432 \mathrm{a}$ \\
\hline 1 & $0.7073 \mathrm{~b}$ & $0.7074 \mathrm{~b}$ & $0.7099 \mathrm{~b}$ \\
\hline 2 & $0.7073 \mathrm{~b}$ & $0.7073 \mathrm{~b}$ & $0.7074 \mathrm{~b}$ \\
\hline 3 & $0.7072 \mathrm{~b}$ & $0.7072 \mathrm{~b}$ & $0.7073 \mathrm{~b}$ \\
\hline 4 & $0.7072 \mathrm{~b}$ & $0.7074 \mathrm{~b}$ & $0.7072 \mathrm{~b}$ \\
\hline 5 & $0.7071 \mathrm{~b}$ & $0.7071 \mathrm{~b}$ & $0.7072 \mathrm{~b}$ \\
\hline 6 & $0.7071 \mathrm{~b}$ & $0.7071 \mathrm{~b}$ & $0.7071 \mathrm{~b}$ \\
\hline
\end{tabular}

Note: Numbers followed by the same letter did not differ significantly at 5\% of DMRT 


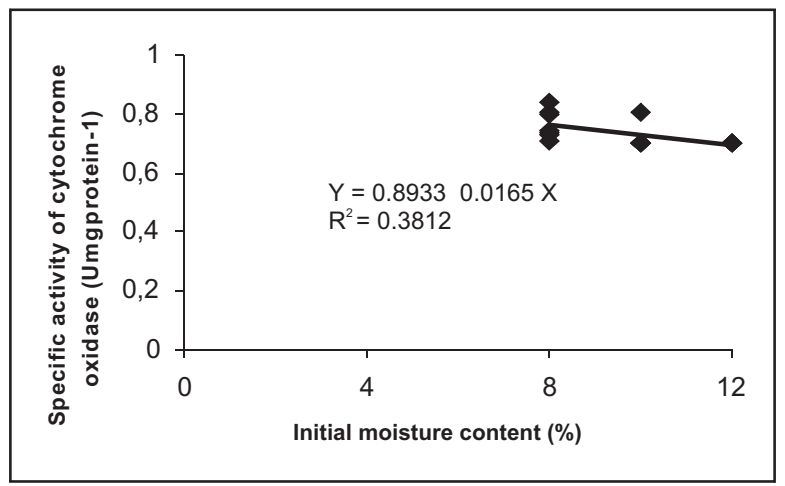

Figure 3. Relationship between initial moisture content and specific activity of cytochrome oxidase

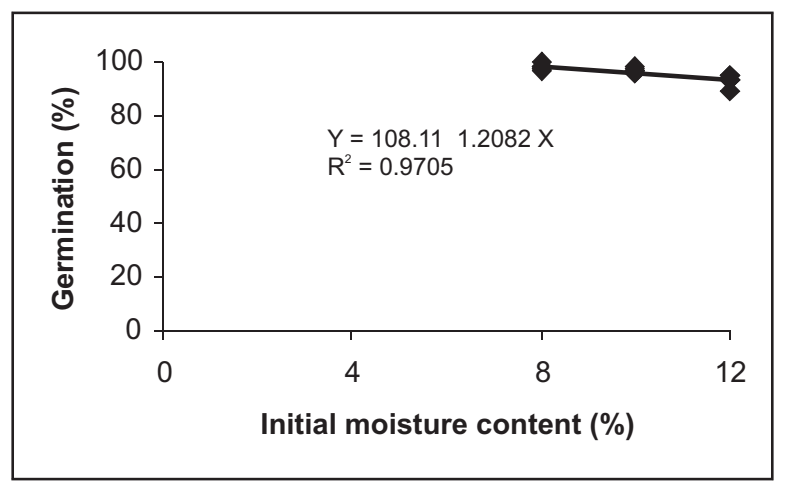

Figure 4. Relationship between initial moisture content and specific activity of seeds and germination

Cytochrome oxidase is an enzyme of mitochondria inner membrane which transfers its electrons to oxygen. It uses the reaction of oxygen and water to move molecular pump. As oxygen is consumed, the energy is stored by pumping hydrogen ions from one side of the membrane to the other. Later, the energy can be used to build ATP (Stryer 1994). On the other hand, cytochrome oxidase acts as enzyme to help add the first electron to oxygen. In general the decrease in succinate dehydrogenase and cytochrome oxidase specific activity in the seeds decreases its respiratory potential so that both the energy (ATP) and food supply for the germinating seeds become less This was shown by deteriorated seeds as presented in Tables 4 and 5. Corn seed deterioration was caused by the decrease of succinate dehydrogenase and cytochrome oxidase activities (Paul \& Mukherji 1976 cit Bewley \& Black, 1982). Activities of succinate dehydrogenase and cytochrome oxidase depend on phospholipids of mitochondria membrane which effect membrane fluidity. Consequently, they affect the mobility of membrane proteins of the mitochondria (Crane \& Sun 1972). The decreases in specific activities of succinate dehydrogenase and cytochrome oxidase cause a decrease in the respiratory rate. 
Table 1. The effect of initial moisture content, packaging materials and storage period on germination $(\%)$

\begin{tabular}{|c|c|c|c|c|}
\hline \multirow{2}{*}{$\begin{array}{c}\text { Initial } \\
\text { moisture } \\
\text { content } \\
(\%)\end{array}$} & \multirow{2}{*}{$\begin{array}{l}\text { Storage } \\
\text { period } \\
\text { (month) }\end{array}$} & \multicolumn{3}{|c|}{ Packaging materials } \\
\hline & & Polyethylene & Wheat & Al.foil \\
\hline \multirow[t]{7}{*}{8} & 0 & $100.00 \mathrm{a}$ & $100.00 \mathrm{a}$ & $100.00 \mathrm{a}$ \\
\hline & 1 & $98.50 \mathrm{a}-\mathrm{d}$ & $98.00 \mathrm{a}-\mathrm{f}$ & $99.25 \mathrm{a}-\mathrm{b}$ \\
\hline & 2 & $97.75 \mathrm{a}-\mathrm{f}$ & $97.50 \mathrm{a}-\mathrm{g}$ & $98.75 \mathrm{a}-\mathrm{c}$ \\
\hline & 3 & $97.75 a-f$ & $97.50 \mathrm{a}-\mathrm{g}$ & $97.75 \mathrm{a}-\mathrm{f}$ \\
\hline & 4 & $97.00 \mathrm{a}-\mathrm{g}$ & $96.00 \mathrm{~b}-\mathrm{i}$ & $97.00 \mathrm{a}-\mathrm{g}$ \\
\hline & 5 & $95.75 \mathrm{~b}-\mathrm{i}$ & $95.50 \mathrm{c}-\mathrm{i}$ & $96.75 \mathrm{a}-\mathrm{h}$ \\
\hline & 6 & $95.50 \mathrm{c}-\mathrm{i}$ & $94.50 \mathrm{e}-\mathrm{i}$ & $96.00 \mathrm{~b}-\mathrm{i}$ \\
\hline \multirow[t]{7}{*}{10} & 0 & $100.00 \mathrm{a}$ & $100.00 \mathrm{a}$ & $100.00 \mathrm{a}$ \\
\hline & 1 & $98.00 \mathrm{a}-\mathrm{d}$ & $98.00 \mathrm{a}-\mathrm{f}$ & $98.50 \mathrm{a}-\mathrm{d}$ \\
\hline & 2 & $97.75 \mathrm{a}-\mathrm{f}$ & $96.75 \mathrm{a}-\mathrm{h}$ & $98.00 \mathrm{a}-\mathrm{f}$ \\
\hline & 3 & $97.75 \mathrm{a}-\mathrm{f}$ & $96.00 \mathrm{~b}-\mathrm{i}$ & $97.00 \mathrm{a}-\mathrm{h}$ \\
\hline & 4 & $97.75 \mathrm{a}-\mathrm{g}$ & $95.25 \mathrm{c}-\mathrm{i}$ & $96.50 \mathrm{~b}-\mathrm{i}$ \\
\hline & 5 & $95.50 \mathrm{c}-\mathrm{i}^{\mathrm{C}}$ & $95.00 \mathrm{c}-\mathrm{i}$ & $95.50 \mathrm{c}-\mathrm{i}$ \\
\hline & 6 & $92.50 \mathrm{i}-\mathrm{k}$ & $92.50 \mathrm{i}-\mathrm{k}$ & $95.25 \mathrm{c}-\mathrm{i}$ \\
\hline \multirow[t]{7}{*}{12} & 0 & $100.00 \mathrm{a}$ & $100.00 \mathrm{a}$ & $100.00 \mathrm{a}$ \\
\hline & 1 & $95.75 \mathrm{~b}-\mathrm{i}$ & 98.00 a-e & $98.25 \mathrm{a}-\mathrm{f}$ \\
\hline & 2 & $95.50 \mathrm{c}-\mathrm{i}$ & $94.75 \mathrm{e}-\mathrm{i}$ & $97.25 \mathrm{a}-\mathrm{g}$ \\
\hline & 3 & $94.50 \mathrm{e}-\mathrm{i}$ & $93.25 \mathrm{~h}-\mathrm{j}$ & $96.50 \mathrm{a}-\mathrm{h}$ \\
\hline & 4 & $94.25 \mathrm{f}-\mathrm{i}$ & $92.75 \mathrm{i}-\mathrm{j}$ & $93.25 \mathrm{~h}-\mathrm{j}$ \\
\hline & 5 & $94.00 \mathrm{~g}-\mathrm{i}$ & $92.75 \mathrm{i}-\mathrm{j}$ & $92.50 \mathrm{i}-\mathrm{k}$ \\
\hline & 6 & $89.25 \mathrm{k}-1$ & 87.751 & $90.75 \mathrm{j}-1$ \\
\hline
\end{tabular}

Note: Numbers followed by the same letter did not differ significantly at $5 \%$ of DMRT

$\mathrm{a}-\mathrm{h}=\mathrm{abcdefgh}$
Table 2. The effect of initial moisture content, packaging materials and storage period on coefficient velocity of germination (CVG)

\begin{tabular}{|c|c|c|c|c|}
\hline \multirow{2}{*}{$\begin{array}{c}\text { Initial } \\
\text { moisture } \\
\text { content } \\
(\%) \\
\end{array}$} & \multirow{2}{*}{$\begin{array}{l}\text { Storage } \\
\text { period } \\
\text { (month) }\end{array}$} & \multicolumn{3}{|c|}{ Packaging materials } \\
\hline & & Polyethylene & Wheat & Al.foil \\
\hline \multirow[t]{7}{*}{8} & 0 & $100.00 \mathrm{a}$ & $100.00 \mathrm{a}$ & $100.00 \mathrm{a}$ \\
\hline & 1 & $98.00 \mathrm{a}-\mathrm{c}$ & $97.50 \mathrm{a}-\mathrm{c}$ & $98.75 \mathrm{a}-\mathrm{b}$ \\
\hline & 2 & $97.50 \mathrm{a}-\mathrm{c}$ & $96.25 \mathrm{~b}-\mathrm{f}$ & $97.75 \mathrm{a}-\mathrm{c}$ \\
\hline & 3 & $96.75 \mathrm{a}-\mathrm{c}$ & $96.00 \mathrm{~b}-\mathrm{g}$ & $97.00 \mathrm{a}-\mathrm{d}$ \\
\hline & 4 & $95.50 \mathrm{~b}-\mathrm{h}$ & $95.50 \mathrm{~b}-\mathrm{h}$ & $97.00 \mathrm{a}-\mathrm{d}$ \\
\hline & 5 & $95.38 \mathrm{c}-\mathrm{h}$ & $95.50 \mathrm{~b}-\mathrm{h}$ & $96.00 \mathrm{~b}-\mathrm{g}$ \\
\hline & 6 & $95.00 \mathrm{c}-\mathrm{i}$ & $91.50 \mathrm{j}-\mathrm{k}$ & $95.00 \mathrm{c}-\mathrm{i}^{\mathrm{B}}$ \\
\hline \multirow[t]{7}{*}{10} & 0 & $100.00 \mathrm{a}$ & $100.00 \mathrm{a}$ & $100.00 \mathrm{a}$ \\
\hline & 1 & $97.63 \mathrm{a}-\mathrm{c}$ & $96.25 \mathrm{~b}-\mathrm{f}$ & $97.75 \mathrm{a}-\mathrm{c}$ \\
\hline & 2 & $97.13 \mathrm{a}-\mathrm{d}$ & $95.63 \mathrm{~b}-\mathrm{h}$ & $97.50 \mathrm{a}-\mathrm{c}$ \\
\hline & 3 & $96.88 \mathrm{a}-\mathrm{d}$ & $95.00 \mathrm{c}-\mathrm{i}$ & $96.13 \mathrm{c}-\mathrm{f}$ \\
\hline & 4 & $96.50 \mathrm{~b}-\mathrm{e}$ & $95.50 \mathrm{~b}-\mathrm{h}$ & $95.75 \mathrm{~b}-\mathrm{h}$ \\
\hline & 5 & $95.50 \mathrm{~b}-\mathrm{h}$ & $95.13 \mathrm{c}-\mathrm{i}$ & $95.50 \mathrm{~b}-\mathrm{h}$ \\
\hline & 6 & $92.50 \mathrm{~h}-\mathrm{j}$ & $92.50 \mathrm{~h}-\mathrm{j}$ & $95.50 \mathrm{~b}-\mathrm{h}$ \\
\hline \multirow[t]{7}{*}{12} & 0 & $100.00 \mathrm{a}$ & $100.00 \mathrm{a}$ & $100.00 \mathrm{a}$ \\
\hline & 1 & 95.63 b-h & $96.00 \mathrm{~b}-\mathrm{h}$ & $97.25 \mathrm{a}-\mathrm{d}$ \\
\hline & 2 & $94.00 \mathrm{~d}-\mathrm{j}$ & $95.25 \mathrm{~d}-\mathrm{j}$ & $96.38 \mathrm{~d}-\mathrm{j}$ \\
\hline & 3 & $93.25 \mathrm{e}-\mathrm{j}$ & $92.75 \mathrm{~g}-\mathrm{j}$ & $95.75 \mathrm{~b}-\mathrm{h}$ \\
\hline & 4 & $93.13 \mathrm{f}-\mathrm{j}$ & $92.75 \mathrm{~g}-\mathrm{j}$ & $93.25 \mathrm{e}-\mathrm{j}$ \\
\hline & 5 & $92.75 g_{-j}$ & $92.00 \mathrm{i}-\mathrm{j}$ & $93.25 e-j$ \\
\hline & 6 & $89.00 \mathrm{k}-\mathrm{l}$ & 87.751 & $89.13 \mathrm{k}-1$ \\
\hline
\end{tabular}

Respiratory rate was thought to depend on continued development of mitochondria (Nakayama et al. 1980). Decrease in respiratory rate caused lowering of the energy flow along the respiratory as well as the proton motive force which in turn will affect the speed of proton conductance. The respiration rate is considered to be controlled by the kinetic properties of the respiratory chain mainly at the level of cytochrome oxidase. Respiration is both controlled by the proton leakage across the inner membrane (Groen et al. 1982) and the respiratory chain (Brand et al. 1988). Respiration is decreased due to the decrease of ADP supply which controls the pathway of proton leakage to other reactions such as the adenine nucleotide and dicarboxylate carrier and cytochrome oxidase (Tager et al. 1983). Respiration within the mitochondria is a function of "unit membranes" and the decrease of mitochondria membrane integrity will change the functional relationships of the membrane-bound components of the respiratory chain. Some scientists reported that mitochondria of older seeds are progressively uncoupled (Wilson \& McDonald 1992). This was related to the decrease of membrane integrity (especially if the transmembrane proton gradient or electron motive force is crucial for coupling). Total $\mathrm{CO}_{2}$ production as index of respiration decreased, but not significantly different (Table 4) indicating that enzymes of the glycolytic pathway and tricarboxylic acid cycle of embryos are not impaired at these stages of deterioration. This process caused polymer synthesis related to energy production (ATP) becomes low. Mitochondria, the primary site of ATP synthesis in seedlings of aged soybean seeds are less efficient 
in producing ATP than mitochondria from seedlings of new seeds (Abu Shakra \& Ching 1967). This may indicate that ATP does not limit the synthetic processes in deteriorated seeds at this stage of germination. Consequently, some other processes, such as decline in activity of specific enzymes, ie. succinate dehydrogenase and cytochrome oxidase, loss of cofactors, etc may occur.

Seed germination and seedling growth are an energy-requiring process and must rely on respiratory metabolism to supply this energy. Thus, a decrease in the rate of respiration of germinating seeds has been shown to precede a decline in the rate of seedling growth (Wilson \& McDonald 1992). Reduced rates of respiration have been associated with seed deterioration in both naturally and artificially aged soybean seeds. Wilson and McDonald (1992) concluded that disruption in mitochondria terminal oxidase, which occurred during artificial ageing of seeds, may lead to an imbalance between glycolytic and tricarboxylic acid cycle or electron transport chain activities resulting in a built up of ethanol and acetaldehyde. Respiratory rates and estimates of mitochondrial functionality were significantly lower in axis tissue of low vigor. Delayed seedling emergence is among the first noticeable symptoms, followed by a slower rate of seedling growth and development and decreased germination. These findings correspond to those reported elsewhere that higher seed moisture content and worst packaging accelerate seed deterioration, causing seed quality losses and therein germinability percentage of stored seeds (Al-Yahya 2001). Soybean seeds have high protein and lipid components (David 1984) and could maintain their germinability only for 3 months under room temperature. Moisture content and oxygen were main causes for lipid oxidation in soybean to lead to rapid seed deterioration and quality decline (Hartman et al. 1994).

In the experiment, it was found that seed moisture content increased and showed negative correlation with germination (Fig. 4) and coefficient velocity of germination (Fig. 5).

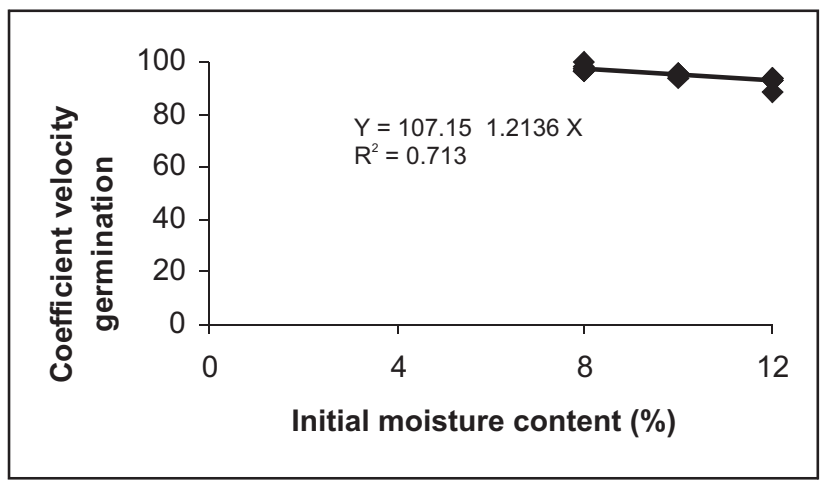

Figure 5. Relationship between initial seed water content and coefficient velocity of germination 
Our results indicated that specific activities of succinate dehydrogenase and cytochrome oxidase decreased, but the viability and vigor as indicated by coefficient velocity of germination were above $80 \%$. Soybean seeds stored at $12 \%$ initial moisture content using aluminium foil bags could retain viability above $80 \%$ for 6 months.

\section{REFERENCES}

Abu Shakra S.S. and T.M. Ching. 1967. Mitochondria activity in germinating new and old soybean seed. Crop Sci., 7:115 118 .

Attuci S., Carde J.P., Raymond P., Saint-Ges V., Speteri A. and A. Pradet. 1991. Oxidative phosphorylation by mitochondria extracted from dry sunflower seeds. Plant physiol., 95:390-398.

Bewley J.D. and M. Black. 1982. Physiology and Biochemistry of Seed in Relation to Germination. Vol. II. Springer-Verlag. Berlin, Heidelberg. New York. 37 p.

Brand M.D., Hafner R.P.a and G.C. Brown. 1988. Control of respiration in non-phosphorylating mitochondria is shared between the proton leak and the respiratory chain. Biochem J., 255:535-539.

Crane F.L. and F.F Sun. 1972. In Electron and Couples Energy Transfer In Biological System. Vol 1. Part 13. Marcel Dekker, New York. p.477-87.

Copeland L.O. and M.B. McDonald. 1985. Principles of Seed Sciences and Technology. Burgress Publishing Company. Minneapolis. Minnesota. p.145-169.

Day D.A. and J.B. Hanson (1977). On methods for isolation of mitochondria from etiolated corn shoots. Plant Sci Lett., 11:99-104.

Groen A. K., Wanders R. J. A., Westerhof H. V., Meer, V. D. and J. M. Tager. 1982. Quatification of the contribution of the various steps to the control of mitochondrial respiration. J. Biol. Chem., 257:27542757.

Harnowo D. and M.M. Adie. 1998. The soybean seed processing and storage technology. Balai Penelitian Tanaman Kacang-kacangan dan Umbi-umbian, Malang. Indonesia. Agris record. p. 80-93.

Hayes M.K., M.H. Luethy and T.E. Elthon. 1991. Mitochondrial Malate Dehydrogenase From Corn. School of Biological Sciences and Centre for Biotechnology, University of Nebraska, Lincoln. International Seed Testing Association. 1996. International rules for seed testing. Seed Sci. and Technol. 27.

Kartono. 2004. Technic of soybean seed var. willis storage at different of moisture content and temperature. Buletin Teknik Pertanian. 9(2): 141-145.

Kozlowsky T.T. 1972. Seed Biology. Vol. III. Academic Press, New York. 569 p.

Lowry O.H., Rosebrough N.J., Farrar A.L. and R.J. Randall. 1951. Protein measurement with the folin phenol reagent. J. Biol. Chem., 193:265-275.

Michael F.M., Karwan, E.K., Kim E. and C. Thomas. 2000. Packaging materials. http://www.eng.buffalo.edu/cowsesl/ces435/food/polymers project.html.

Moller I.M. and W. rein. 1986. Membrane bound NADPH dehydrogenase in plant cell. Annu. Rev. Plant Physiol., 37:309-334.

Nakayama N., Sugimoto N.I. and T. Asahi. 1980. Presence in dry cotyledons of soluble succinate dehydrogenase that is assembled into the mitochondrial inner membrane suring seed imbibitions. Plant Physiol., 65:229-233.

Nawa Y. and T. Asahi. 1971. Biochemical study on the development of mitochondria in pea cotyledons during the early stage of germination. Plant Physiol., 51:833-838.

Pessu O., Adindu M.N. and O.C. Umeozor. 2005. Effect of long term storage on the quality of soybean, Glycine $\max (\mathrm{L})$. Merrill in Different containers in Southern Nigerian. Global Journal of Pure and Applied Sciences. 11(2):165-168. 
Effect of initial moisture content, packaging and storage period - Aurelia Tatipata

Sadjad S. 1980. Panduan Mutu Benih Tanaman Kehutanan di Indonesia IPB. Bogor. 205p.

Statistical Analysis System. 1986. SAS/STAT User's Guide Version 6.01. SAS Institute Inc. Cary NC.

Singer T.P., Oesreicher G. and P. Hogue. 1972. Regulation of succinate dehydrogenase in higher plants. I. Some General Characteristics of The Membrane-bound Enzyme. Plant Physiol., 53:616-621.

Stryer L. 1994. Biochemistry. $4^{\text {th }}$ Ed. Stanford University. New York: H. Freeman Company. 1065 p.

Sudarmadji S., Haryono B. and Suhardi. 1984. Prosedur Analisa Untuk Bahan Makanan dan Pertanian. Fakultas Teknologi Pertanian. Universitas Gajah Mada. Penerbit Liberty. Yogyakarta.

Sumarno and Harnoto. 1983. Padi dan palawija. Proc. Sem. Balittan Bogor. 2: 27-28. Pusat Penelitian dan Pengembangan Pertanian. Bogor.

Tager J.M., Groen A.K., Wanders R.J.A., Duszynski J., Westerhof H.V. and R.C.Veryoon. 1983. Control of mitochondrial respiration. Biochem. Soc. Trans., 11:40-43.

Wilson D.O. Jr. and M.B. Jr. McDonald. 1992. Mechanical damage in bean (Phaseolus vulgaris L.) seed in mechanized and non-mechanized threshing systems. Seed Sci. and Technol., 20:571-582. 